OPEN ACCESS

Edited by:

Lei Deng,

Central South University, China

Reviewed by:

Xiaowen Chen,

Harbin Medical University, China

Jie Zhang,

Tongji University, China

*Correspondence:

Dekai Zhang

dekaiz@hotmail.com

Xinhong Wang

yuner1976@126.com

${ }^{\dagger}$ These authors have contributed equally to this work

Specialty section:

This article was submitted to

Molecular Medicine

a section of the journal

Frontiers in Cell and Developmental

Biology

Received: 03 May 2020

Accepted: 29 June 2020

Published: 21 July 2020

Citation:

Liu J, Wang Y, Chu Y, Xu R,

Zhang $D$ and Wang $X$ (2020)

Identification of a TLR-Induced

Four-IncRNA Signature as a Novel

Prognostic Biomarker in Esophageal

Carcinoma

Front. Cell Dev. Biol. 8:649.

doi: 10.3389/fcell.2020.00649

\section{Identification of a TLR-Induced Four-IncRNA Signature as a Novel Prognostic Biomarker in Esophageal Carcinoma}

\author{
Jing Liu't, Yanbo Wang ${ }^{2 t}$, Yanjie Chu' ${ }^{1}$, Ruiling Xu' ${ }^{1}$, Dekai Zhang ${ }^{3 *}$ and Xinhong Wang ${ }^{1 *}$ \\ ${ }^{1}$ Department of Gastroenterology and Hepatology, The Second Affiliated Hospital of Harbin Medical University, Harbin, \\ China, ${ }^{2}$ Department of Thoracic Surgery, Harbin Medical University Cancer Hospital, Harbin, China, ${ }^{3}$ Center for Infectious \\ and Inflammatory Diseases, Texas A\&M University, Houston, TX, United States
}

Long non-coding RNAs (IncRNAs) have emerged as key regulators of Toll-like receptor (TLR) signaling to control innate immunity, and this regulatory mechanism has recently been implicated in esophageal carcinoma (ESCA). However, a comprehensive analysis of TLR-induced IncRNAs and their roles in diagnosis and prognosis in ESCA is still lacking. In this study, we first investigated the precise relationship between IncRNA perturbations and alteration of TLR signaling by constructing the IncRNA-TLRs coexpression network involved in ESCA, and identified 357 TLR-related IncRNAs. Of them, four TLR-related IncRNAs (AP000696.1, LINC00689, LINC00900, and AP000487.1) are significantly associated with the overall survival (OS) of ESCA patients, and utilizing this four-IncRNA signature is capable of stratifying patients into high-risk and low-risk groups with significantly different OS in the discovery set. Further analysis in different independent patient sets also confirmed the robustness of the prognostic value of the four-TLR-IncRNA signature in predicting the OS of ESCA patients. Moreover, the results of multivariate analysis in different patient sets indicated that the four-TLR-IncRNA signature is an independent factor after adjusted by other clinical factors. Thus, we have identified a TLR-induced four-IncRNA signature, which represents a promising prognosis biomarker for ESCA, and our study might provide new candidate targets for therapeutic intervention via targeting TLR-induced IncRNAs in ESCA patients.

Keywords: Toll-like receptor, long non-coding RNAs, esophageal carcinoma, signature, biomarker

\section{INTRODUCTION}

Esophageal carcinoma (ESCA) is a common type of malignant tumor in the digestive system. Two major pathologic subtypes of ESCA are esophageal adenocarcinoma and esophageal squamous cell carcinoma (ESCC; Napier et al., 2014; Jain and Dhingra, 2017). There were an estimated 18,440 newly diagnosed cases and 16,170 deaths of ESCA, which account for approximately $1 \%$ of all diagnosed cancer cases and $2.7 \%$ of all cancer-related deaths in the United States (Siegel et al., 2020). Although ESCA could be treated by esophagectomy in combination with chemotherapy and radiation therapy, the outcome of ESCA is generally tended to be relatively poor, and the 5 -year relative survival rate is approximately $20 \%$ due to the late 
diagnosis (Mawhinney and Glasgow, 2012; D’Journo and Thomas, 2014; Rustgi and El-Serag, 2014; Siegel et al., 2020). TNM staging system was most commonly used for guiding clinical decision making but is still insufficient for improving ESCA diagnosis and prognosis because of intrinsically molecular heterogeneity of ESCA (The Cancer Genome Atlas Research Network, 2017; Liu et al., 2017). Therefore, molecular biomarkers were urgently needed for complementing the TNM staging system and providing more precise prediction and consequently improved personalized cancer care.

Toll-like receptors (TLRs), one class of pattern-recognition receptors (PRRs), play crucial roles in the innate immune system by recognizing pathogen-associated molecular patterns (Kawasaki and Kawai, 2014). It has been suggested that TLR-mediated inflammation promotes tumor growth and development (Cen et al., 2018). TLRs have also been found to be involved in the tumor cell death by inducing apoptosis, autophagy, and programmed necrosis in tumor cells (Cen et al., 2018). Members of the TLR family, including ten TLRs, have been discovered in humans, and it has been reported that some of them are up-regulated in many tumor cells, tissues, or tumor cell lines (So and Ouchi, 2010). For example, TLR3, TLR4, TLR7, and TLR9 have significantly higher expression in ESCA samples compared to normal tissues, which are associated with poor prognosis and lymph node metastasis (Sheyhidin et al., 2011).

Large-scale genomic and transcriptome analyses have suggested that less than $3 \%$ of the human genome encodes proteins and at least $75 \%$ are actively transcribed to noncoding RNA (ncRNA) (Djebali et al., 2012; Li and Liu, 2019). According to sequence length, ncRNA are generally divided into small ncRNAs with size $<200$ nucleotides and long ncRNA (lncRNA) larger than 200 nucleotides in length (Han $\mathrm{Li}$ and Chen, 2015). A large number of functional studies have shown that lncRNAs play critical roles in a variety of cellular processes by regulating gene expression through diverse mechanisms at transcriptional, post-transcriptional and epigenetic levels (Rinn and Chang, 2012; Perry and Ulitsky, 2016; Koch, 2017). Furthermore, altered lncRNAs expression has been widely discovered in various cancers and have been used as novel biomarkers for cancer diagnosis and prognosis (Prensner and Chinnaiyan, 2011; Qiu et al., 2013; Zhou et al., 2018a; Bao et al., 2019; Sun et al., 2020). Increasing evidence indicated that lncRNA is emerging a key regulator of TLR signaling and innate immunity (Murphy and Medvedev, 2016; Zhang et al., 2020; Zhou et al., 2020), altered lncRNAs expression mediated via control of TLR signaling have been implicated in ESCA (Tang et al., 2015). However, a comprehensive analysis of lncRNAs changes induced by TLRs and their roles in diagnosis and prognosis in ESCA is lacking.

In the present study, we tried to investigate the precise relationship between lncRNA perturbations and TLRs dysfunction by constructing the IncRNA-TLRs co-expression network involved in ESCA. Through integrative analysis of transcriptome data and clinical data, we identified and validated a four-lncRNA signature induced by TLRs for improving outcome prediction of ESCA patients.

\section{MATERIALS AND METHODS}

\section{Clinical and Molecular Profiles Data of ESCA Patients}

Clinical and lncRNA expression profile of 179 ESCA patients and paired 179 normal tissues profiled by Agilent-038314 CBC Homo sapiens IncRNA + mRNA microarray V2.0 were obtained from the Gene Expression Omnibus (GEO) database (the accession number is GSE53625) ${ }^{1}$ ( $\mathrm{Li}$ et al., 2014). All 177 ESCA patients with survival information $>1$ month were randomly divided into the discovery set $(n=120)$ for biomarker identification and the internal testing set for validation $(n=57)$ according to the ratio of 2:1 (Hartwell et al., 2018; Penn-Nicholson et al., 2019; Garrido et al., 2020). Clinical and RNA-seq data of another ESCA patient set (including 160 with survival information) was obtained from The Cancer Genome Atlas (TCGA) database ${ }^{2}$ for the independent validation. The clinical characteristics of ESCA patients in each dataset were shown in Table 1.

\section{TLR Genes}

A total of 104 TLR-related genes were obtained from the TLR signaling pathways in Kyoto Encyclopedia of Genes and Genomes (KEGG) database.

\section{Processing and Analysis of IncRNA Expression Data}

The lncRNA+mRNA array data were analyzed for data summarization, quantile normalization and quality control by using the GeneSpring software V11.5 (Agilent). Then probe sequence was aligned to lncRNA sequence of GENCODE using the blast software and obtained 6850 lncRNAs for further analysis. RNA-seq data of TCGA ESCA patients were reannotated based on the IncRNA annotations in GENCODE, and obtained 15,873 lncRNAs.

Differential expression analysis for lncRNAs was performed between 179 paired ESCA and normal tissues using the R package "limma." Those lncRNAs with $\mid \log 2$ (fold change) $\mid>1$ and false discovery rate $($ FDR) adjusted $p$-value $<0.05$ were considered as significantly differentially expressed lncRNAs (Zhou et al., 2018a). Hierarchical clustering analysis was performed with the R package "pheatmap" using manhattan distance and "ward.D” method.

\section{Function Enrichment Analysis}

Function enrichment analysis of mRNAs was performed with the R package "clusterProfiler" which can implement the statistical analysis and visualization of functional profiles for genes and gene clusters (Yu et al., 2012).

\section{Statistical Analysis}

The relationship between expression levels of lncRNAs and overall survival (OS) were evaluated using the univariate and multivariate Cox regression analysis. LncRNA biomarkers were

\footnotetext{
${ }^{1}$ https://www.ncbi.nlm.nih.gov/geo/query/acc.cgi?acc=GSE53625

${ }^{2}$ https://www.cancer.gov/
} 
TABLE 1 | Clinical characteristics of ESCA patients in each dataset.

\begin{tabular}{|c|c|c|c|}
\hline Covariates & $\begin{array}{l}\text { Discovery set } \\
\quad(n=120)\end{array}$ & $\begin{array}{l}\text { Internal testing } \\
\text { set }(n=57)\end{array}$ & $\begin{array}{l}\text { TCGA set } \\
(n=160)\end{array}$ \\
\hline Age, years (mean $\pm S D$ ) & $59.9 \pm 12.1$ & $57.8 \pm 8.8$ & $62.1 \pm 11.9$ \\
\hline \multicolumn{4}{|l|}{ Gender, no (\%) } \\
\hline Male & $102(85.0)$ & $43(75.4)$ & $137(85.6)$ \\
\hline Female & $18(15.0)$ & $14(24.6)$ & $23(14.4)$ \\
\hline \multicolumn{4}{|l|}{ Stage, no (\%) } \\
\hline । & $6(5.0)$ & $3(5.3)$ & $16(10.0)$ \\
\hline$\|$ & $48(40.0)$ & $28(49.1)$ & $68(42.5)$ \\
\hline III & $66(55.0)$ & $26(45.6)$ & 49 (30.6) \\
\hline IV & $0(0.0)$ & $0(0.0)$ & $8(5.0)$ \\
\hline Unknown & $0(0.0)$ & $0(0.0)$ & $19(11.9)$ \\
\hline \multicolumn{4}{|l|}{ Alcohol use, no (\%) } \\
\hline Yes & $72(0.6)$ & 33 (57.9) & $111(69.4)$ \\
\hline No & $48(0.4)$ & $24(42.1)$ & $46(28.7)$ \\
\hline Unknown & $0(0.0)$ & $0(0.0)$ & $3(1.9)$ \\
\hline \multicolumn{4}{|l|}{ Pathological T, no (\%) } \\
\hline $\mathrm{T} 1$ & $7(5.8)$ & $5(8.8)$ & 27 (16.9) \\
\hline T2 & $14(11.7)$ & $11(19.3)$ & $37(23.1)$ \\
\hline Т3 & 76 (63.3) & $34(59.6)$ & $76(47.5)$ \\
\hline T4 & $23(19.2)$ & 7 (12.3) & $4(2.5)$ \\
\hline Unknown & $0(0.0)$ & $0(0.0)$ & $16(10.0)$ \\
\hline \multicolumn{4}{|l|}{ Pathological $N$, no (\%) } \\
\hline NO & $54(45.0)$ & $28(49.1)$ & $65(40.6)$ \\
\hline N1 & $41(34.2)$ & $20(35.1)$ & $63(39.4)$ \\
\hline N2 & $18(15.0)$ & $4(7.0)$ & $9(5.6)$ \\
\hline N3 & $7(5.8)$ & $5(8.8)$ & $6(3.8)$ \\
\hline Unknown & $0(0.0)$ & $0(0.0)$ & 26 (10.6) \\
\hline \multicolumn{4}{|l|}{ Vital status, no (\%) } \\
\hline Alive & $48(40.0)$ & 25 (43.9) & 97 (60.6) \\
\hline Dead & $72(60.0)$ & $32(56.1)$ & $63(39.4)$ \\
\hline
\end{tabular}

identified using the least absolute shrinkage and selection operator (LASSO) method. The lncRNAs-based signature was constructed using the linear sum of expression levels of lncRNAs biomarkers and the weights derived from multivariate Cox regression analysis (Zhou et al., 2018b; Zhou et al., 2018c). The optimal risk score was defined using the R package "maxstat." Kaplan-Meier estimate and the log-rank test were used to compare survival differences between high-risk group and lowrisk group. The prognostic value of signature was assessed using time-dependent receiver-operating characteristic (ROC) analysis. All statistical analyses were performed with R software (version 3.6.3). ${ }^{3}$

\section{RESULTS}

\section{Identification of TLR-Related IncRNAs in ESCA}

We first tried to identify the lncRNAs associated with ESCA. Expression profiles of lncRNAs between 179 paired ESCA

${ }^{3}$ www.r-project.org patients and normal tissues were compared to find differentially expressed lincRNAs from data of RNA-seq or microarray by performing analyses with the $\mathrm{R}$ software package bioconductor "limma" (Zhou et al., 2018a). We identified a total of 587 lncRNAs with significant difference (| log2(fold change)| $>1$ and FDR adjusted $p$-value $<0.05)$. Among them, 258 lncRNAs were found to be up-regulated and 329 lncRNAs to be down-regulated in ESCA (Figure 1A and Supplementary Table 1). Hierarchical clustering analysis suggested that the expression pattern of these 587 lncRNAs can significantly distinguish between ESCA patients and normal tissues (chisquare test $p<2.2 \mathrm{e}-16$ ) as shown in Figure 1B. Then we examined the correlation between expression levels of 104 TLR-related genes and 587 differentially expressed lncRNAs by calculating the Pearson correlation coefficient. Finally, 357 lncRNAs were significantly correlated with that of at least one of TLR genes (Pearson correlation coefficient $>0.6$ and $p<0.05)$ and were defined as TLR-related lncRNAs. As shown in Figure 1C, we constructed a TLR-related lncRNAs-mRNA network which included 1404 edges involved in 51 TLR genes and 357 lncRNAs.

\section{Identification of a Four-IncRNA Signature Induced by TLRs in the Discovery Set}

To identify potential TLR-related lncRNA biomarkers for predicting OS, we performed feature selection for 357 lncRNAs in the TLR-related lncRNAs-mRNA network in the discovery set, and identified four TLR-related lncRNAs (AP000696.1, LINC00689, LINC00900, and AP000487.1) as optimal biomarkers, which were significantly associated with OS of ESCA patients (Table 2). Then these four TLR-related lncRNAs biomarkers were integrated into a signature using the linear sum of expression levels of lncRNAs biomarkers and the weights derived from multivariate Cox regression analysis as follows: four TLR-related lncRNAs signature (four-TLR-lncRNA signature $)=(0.239 \times$ expression level of AP000696.1 $)+(-0.240 \times$ expression level of LINC00689 $)+$ $(0.124 \times$ expression level of LINC00900) $+(0.239 \times$ expression level of AP000487.1). The optimal risk cutoff value of the four-TLR-lncRNA signature was determined using the $\mathrm{R}$ package "maxstat" in the discovery set. The optimal risk cutoff value of four-TLR-lncRNA could stratify 120 patients into the high-risk group $(n=88)$ and low-risk group $(n=32)$ with significantly different OS (Log-rank test $p<0.001$ ) (Figure 2A). As shown in Figure 2A, the low-risk patients have a better OS (median 4.93 years) than those with high-risk (median 1.56 years). The five- survival rate of patients in the low-risk group is $49.5 \%$, which is higher than those in the high-risk group (12.5\%). The AUC for the four-TLR-lncRNA signature prognostic model was 0.77 at five years and 0.67 at three years of OS (Figure 2B). The distribution of risk scores, the survival status and lncRNA expression of patients were ranked by risk score and were shown in Figure 2C. As shown in Figure 2C, three lncRNAs (AP000696.1, LINC00900, and AP000487.1) are associated with high-risk and are upregulated expressed in high-risk patients, and one lncRNAs 

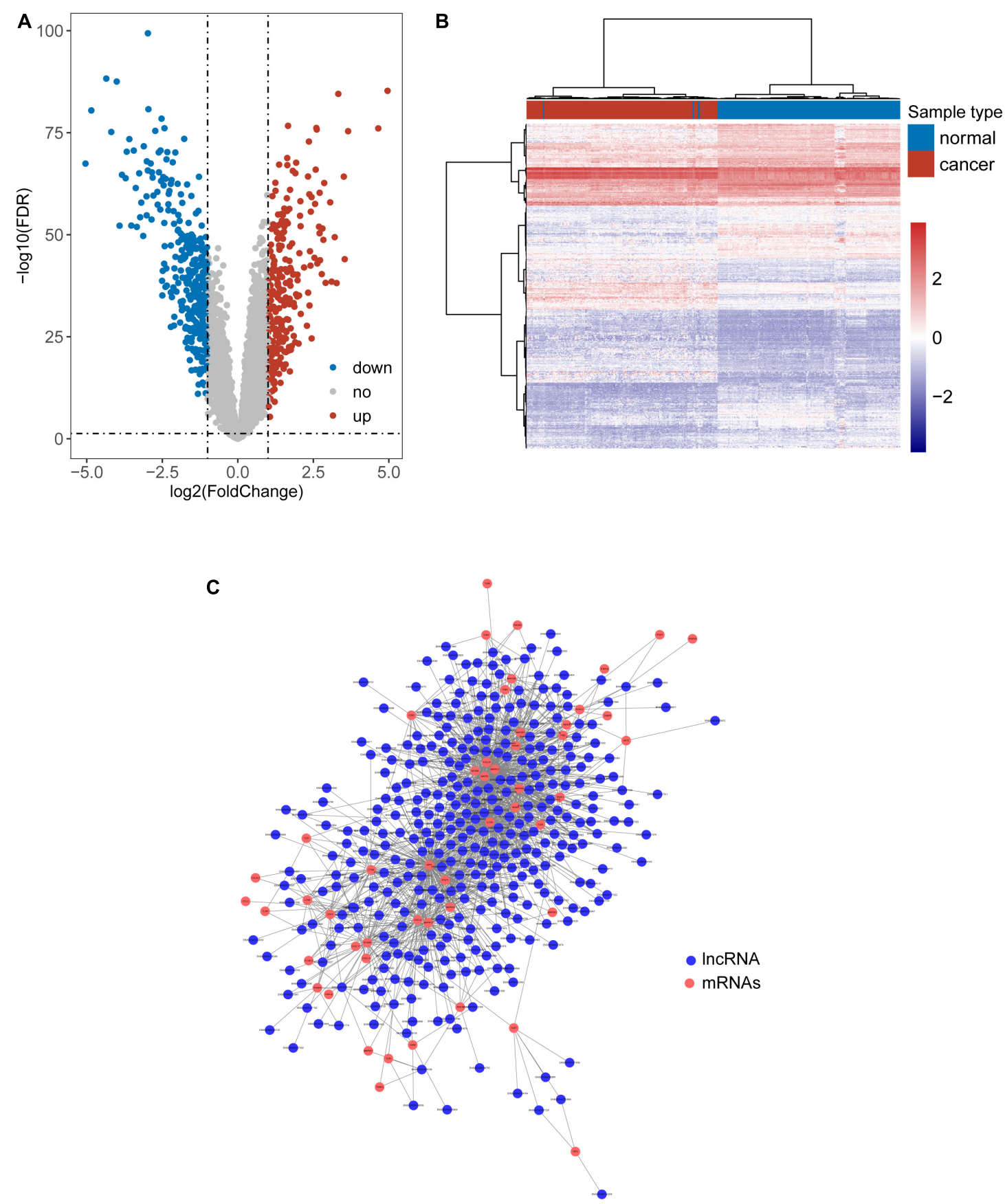

FIGURE 1 | Identification of Toll-like receptors-induced IncRNAs in ESCA. (A) Volcano Plots of differentially expressed IncRNAs. (B) Heatmap of hierarchical clustering analysis based on differentially expressed IncRNAs. (C) The global view of a TLR-related IncRNAs-mRNA network.

TABLE 2 | Four TLR-related IncRNA biomarkers significantly associated with the overall survival in the discovery patient set.

\begin{tabular}{lcccc}
\hline Ensembl id & Gene name & Genomic location & HR & $\mathbf{9 5} \% \mathbf{C l}$ \\
\hline ENSG00000231324 & AP000696.1 & Chr 21: 36,632,681-36,637,033(-) & 1.36 & $1.147-1.614$ \\
ENSG00000231419 & LINC00689 & Chr 7: 159,006,522-159,030,195(+) & 0.637 & $0.44-0.923$ \\
ENSG00000246100 & LINC00900 & Chr 11: 115,753,889-115,760,646(-) & 1.277 & $1.07-1.524$ \\
ENSG00000246889 & AP000487.1 & Chr 11: 70,372,246-70,398,488 (-) & 1.039 & $1.039-1.633$
\end{tabular}



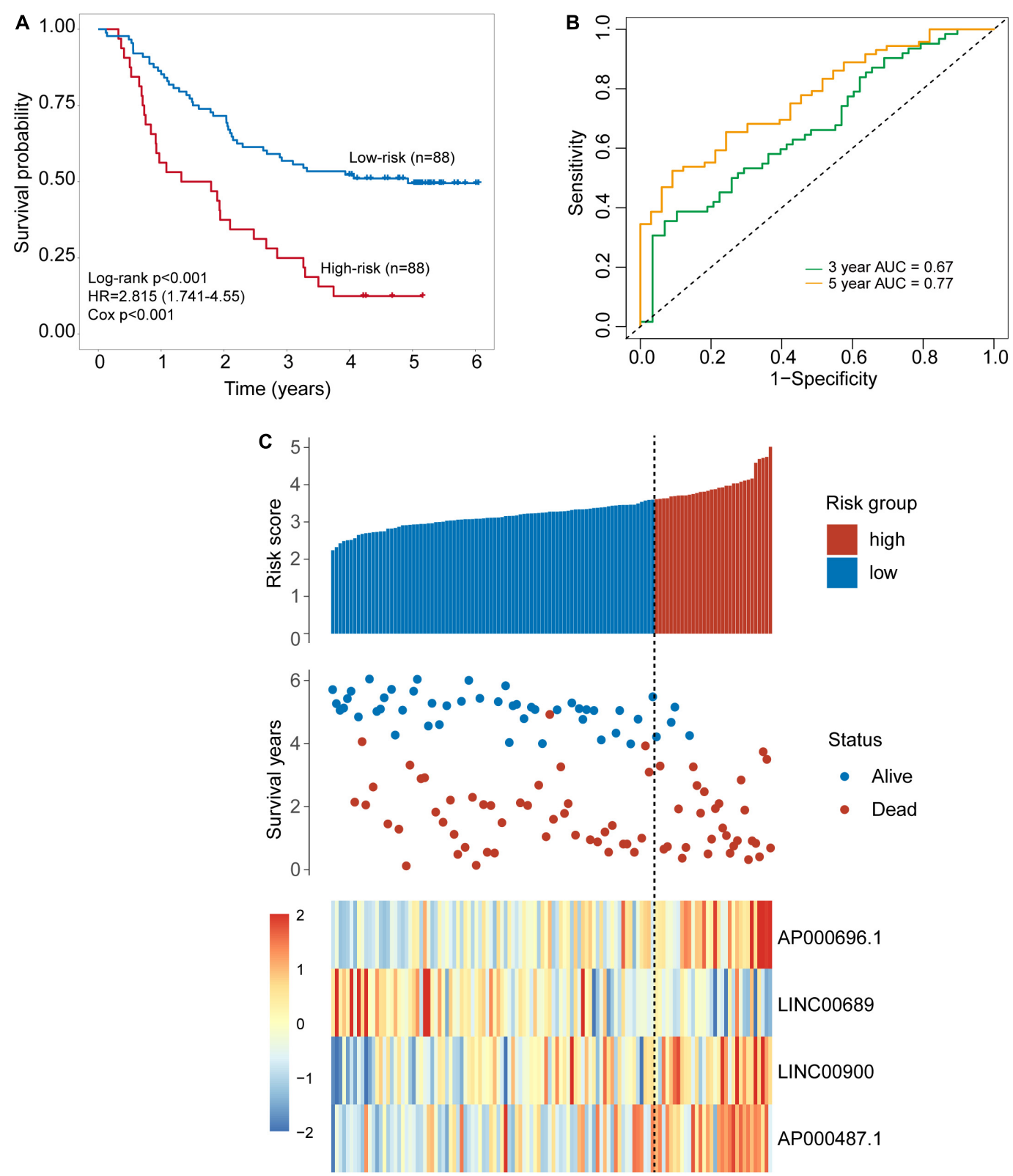

FIGURE 2 | Development of a four-IncRNA signature induced by Toll-like receptors in the discovery set. (A) Kaplan-Meier survival curves of overall survival between the high-risk group and low-risk group stratified by four-IncRNAs signature. (B) The 3- and 5-year time-dependent ROC analysis. (C) Distribution of risk scores, patients' survival status and IncRNAs expression pattern.

(LINC00689) tended to be a protective factor and is up-regulated in the low-risk group.

\section{Validation of the Four-TLR-IncRNA Signature in the Internal Testing Set}

The same score formula and risk cutoff value obtained from the discovery set were applied to the patients in the internal testing set and calculate the risk score for each patient. With the fourTLR-lncRNA signature, patients of the internal testing set were classified into the high-risk group $(n=9)$ and low-risk group $(n=48)$. As shown in Figure 3A, the survival time of the highrisk group patients was significantly shorter than that of low-risk group patients (median survival 1.34 years vs. 4.69 years, logrank test $p=0.054$ ) (Figure 3A). The five-survival rate of patients in the low-risk group is $49.2 \%$, whereas the corresponding fivesurvival rate of patients in the high-risk is $22.2 \%$. The AUC for the four-TLR-lncRNA signature prognostic model was 0.56 at five years and 0.55 at three years of OS (Figure 3B). The distribution of risk scores, the survival status and lncRNA expression of patients were ranked by risk score and were shown in Figure 3C. As observed in the discovery set, the expression patterns of 

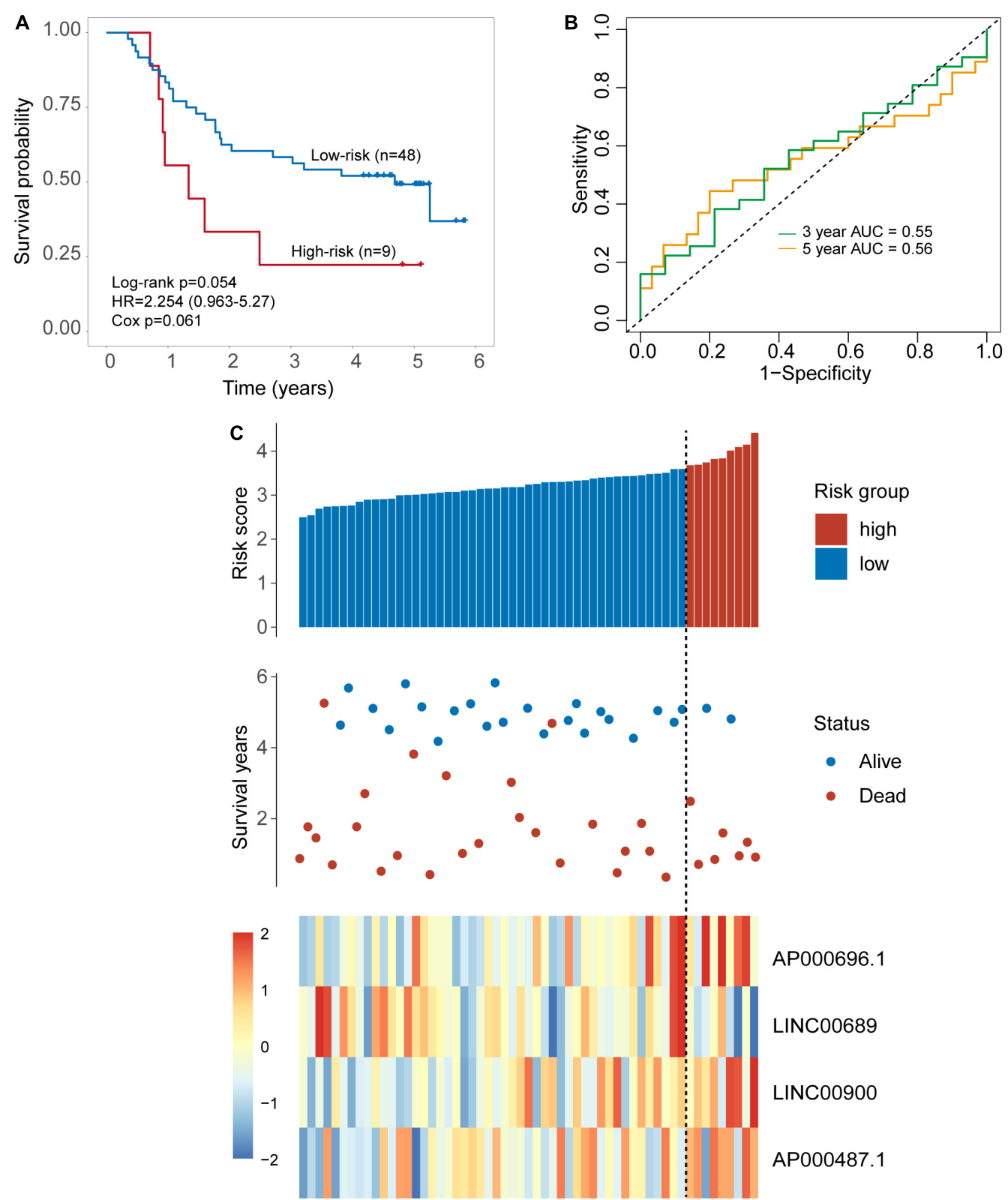

FIGURE 3 | Validation of the TLR-induced four-IncRNA signature in the internal testing set. (A) Kaplan-Meier survival curves of overall survival between the high-risk group and low-risk group stratified by a four-IncRNAs signature. (B) The 3- and 5-year time-dependent ROC analysis. (C) Distribution of risk scores, patients' survival status and IncRNAs expression pattern.

four TLR-related lncRNAs biomarkers are consistent with that in the discovery set. Three lncRNAs (AP000696.1, LINC00900, and AP000487.1) are risk factors, whereas the lncRNAs LINC00689 is a protective factor.

\section{Independent Validation of the Four-TLR-IncRNA Signature in the TCGA Set With Cross-Platform}

To further examine the robustness of the four-TLR-lncRNA signature in predicting OS, we tested the prognostic value of the
four-TLR-lncRNA signature in another completely independent TCGA set with RNA-seq platform. When the four-TLR-lncRNA signature was applied to the TCGA set, the optimal risk cutoff value classified 160 patients into the high-risk group $(n=94)$ and low-risk group $(n=66)$. As shown in Figure 4A, there is a significant difference in OS between high-risk and lowrisk groups. As in the discovery and internal testing sets, patients in the high-risk group had significantly shorter OS (median 2.09 years) than those in the low-risk group (median 3.73 years). The five-survival rate of patients in the low-risk group is $33.1 \%$, whereas the corresponding five-survival rate 
of patients in the high-risk is $6.6 \%$. The AUC for the fourTLR-lncRNA signature prognostic model was 0.72 at five years and 0.70 at three years of OS (Figure $4 \mathbf{B}$ ). The distribution of risk scores, the survival status and lncRNA expression of patients were ranked by risk score and were shown in Figure 4C. As observed in the discovery and internal testing sets, the expression patterns of four TLR-related lncRNAs biomarkers are consistent with that in the discovery and internal testing sets. Three IncRNAs (AP000696.1, LINC00900 and
AP000487.1) are risk factors, whereas the lncRNAs LINC00689 is a protective factor.

\section{Prognostic Value of the Four-TLR-IncRNA Signature Is Independent of Clinical Factors}

We next performed the univariate and multivariate analysis with the four-TLR-lncRNA signature and other clinical factors
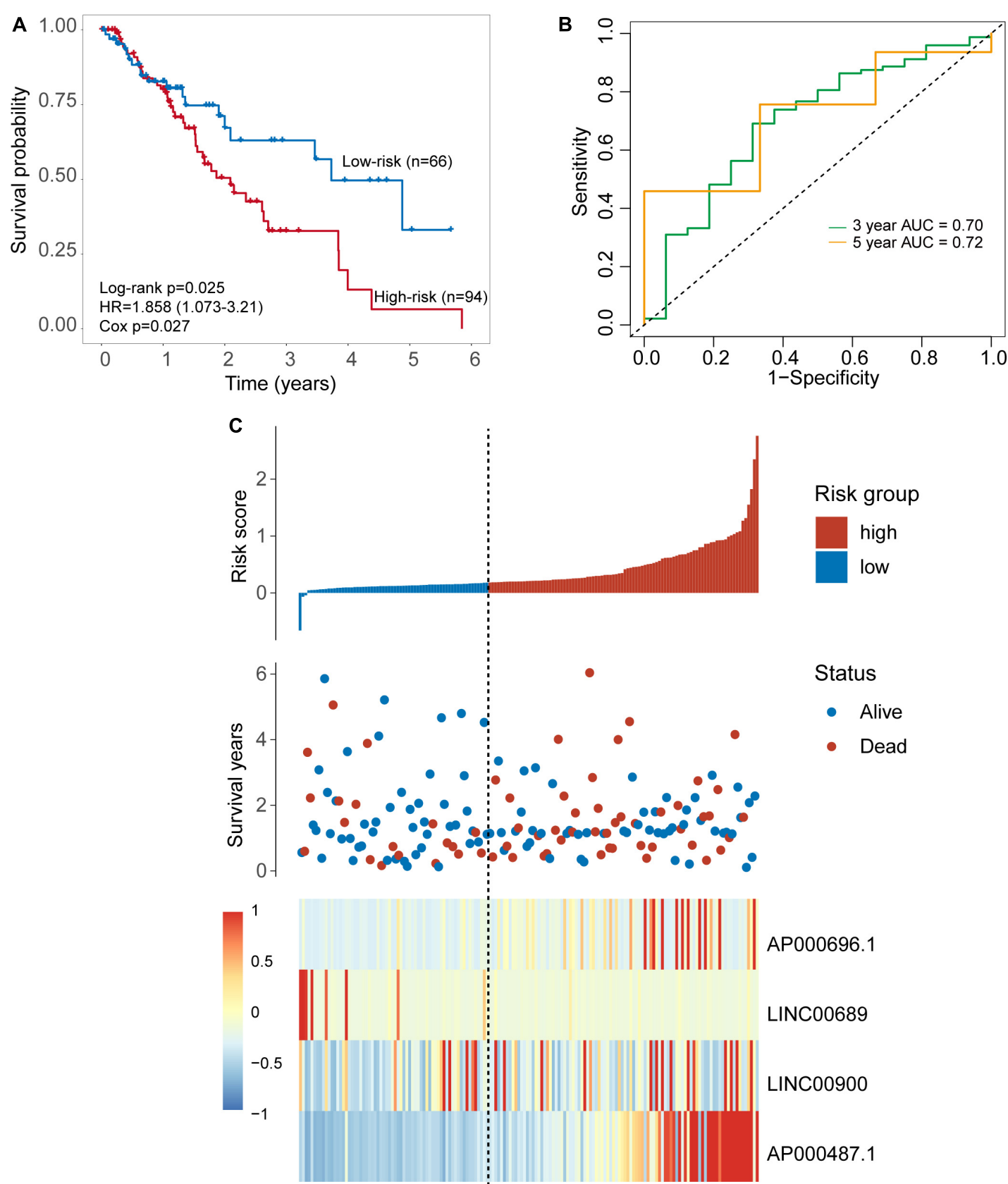

FIGURE 4 | Further validation of the TLR-induced four -IncRNA signature in the TCGA set. (A) Kaplan-Meier survival curves of overall survival between the high-risk group and low-risk group stratified by four-IncRNAs signature. (B) The 3- and 5-year time-dependent ROC analysis. (C) Distribution of risk scores, patients' survival status and IncRNAs expression pattern. 
(including age, gender, stage and alcohol) to examine whether the survival prediction ability of the four-TLR-lncRNA signature is independent of other clinical factors in three patient sets. In the discovery set, although univariate analysis revealed that the four-TLR-lncRNA signature $(p<0.001)$, age $(p=0.012)$, and gender $(p=0.07)$ were all significantly or marginally significantly associated with OS, the four-TLR-lncRNA signature $(p<0.001)$ and age $(p=0.003)$ were significant in the multivariate analysis. In the independent TCGA testing set, the four-TLR-lncRNA signature $(p=0.034)$ and stage $(p=0.017$ and $<0.001)$ were independent prognostic factors in the multivariate analysis (Table 3).

\section{Functional Analysis of the Four-TLR-IncRNA Signature}

We first examined the correlation between expression levels of each of four TLR-related lncRNA biomarkers and mRNAs using the Pearson correlation coefficient and identified 3313
mRNAs related to lncRNA biomarkers. Of them, 22 mRNAs are well-known TLR genes. The results of the hypergeometric test revealed that TLR genes were marginally significantly enriched in mRNAs co-expressed with lncRNA biomarkers (hypergeometric test $p=0.076$ ) (Figure 5A). We further performed functional enrichment analysis of GO and KEGG for 3313 mRNAs related to lncRNA biomarkers and selected top 15 enriched GO terms and KEGG pathways, which were shown in Figure 5B. We found that mRNAs co-expressed with lncRNA biomarkers are enriched in TLR-related and cancer-related GO terms and KEGG pathways such as ECM-receptor interaction, Focal adhesion and PI3K-Akt signaling pathway.

\section{DISCUSSION}

In addition to traditional treatments, including esophagectomy followed by chemotherapy and radiation therapy, other treatment options for ESCA patients continue to evolve, such

TABLE 3 | Univariate and Multivariate Cox Regression Analysis of Overall Survival in each patient set.

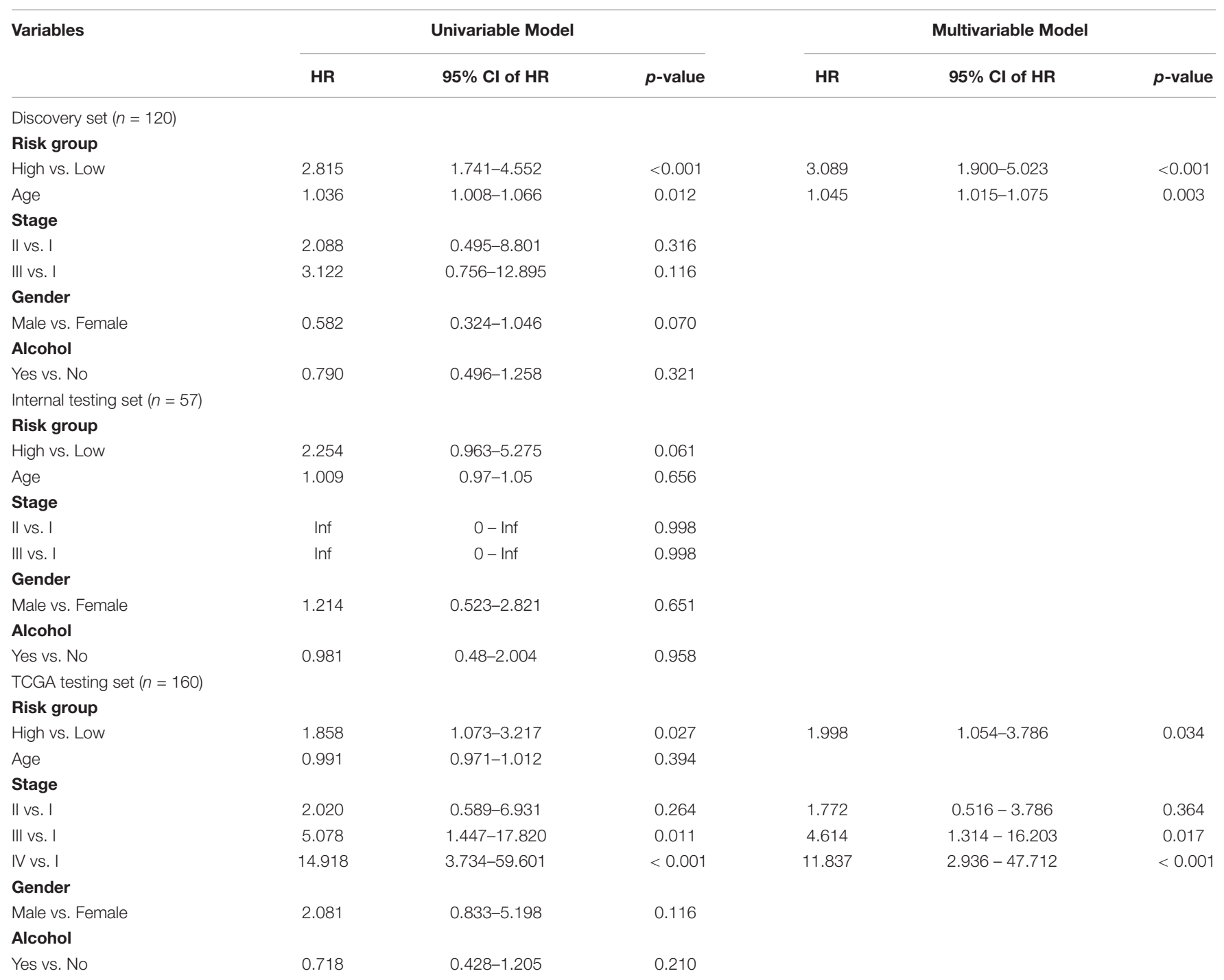




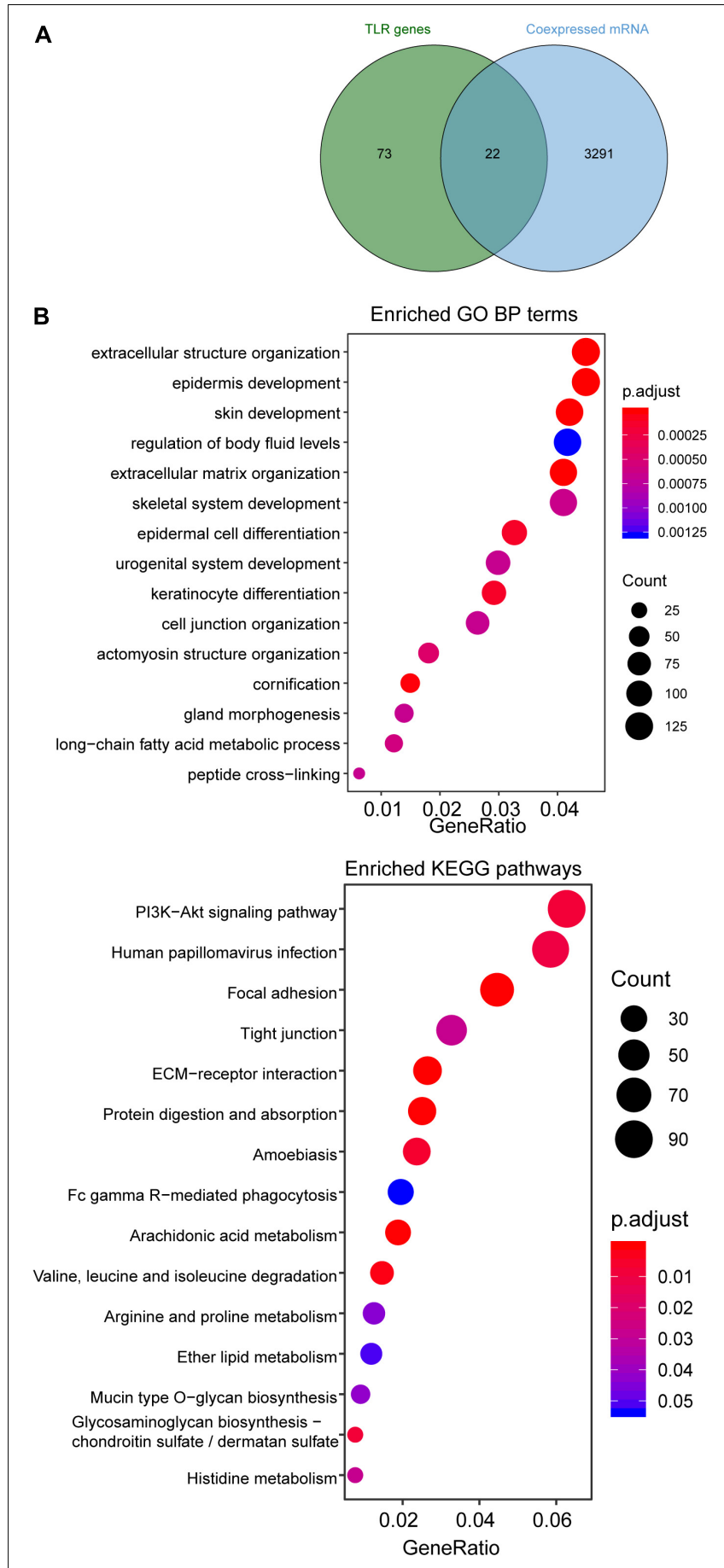

FIGURE 5 | Functional enrichment analysis. (A) Venn diagram of co-expressed genes with IncRNAs and known TLR genes. (B) Enriched GO terms and KEGG pathways.

as targeted drug therapy and immunotherapy (Wald et al., 2017). However, most ESCA patients are still faced with a poor prognosis with a five-year relative survival rate of about $20 \%$. With considerable progress in our understanding of molecular characteristics of ESCA, it is now known that ESCA is a heterogeneous disease characterized by different molecular features associated with varied outcomes. Therefore, molecular profiles, including DNA, RNA, or proteins, have been proven to be a promising marker for improving clinical decisionmaking for diagnosis and prognosis of ESCA patients (Tanzer et al., 2013). LncRNAs have been found to be expressed in the cell and/or tissue/tumor-specific manner, highlighting their emerging roles as novel molecular markers in various cancers (Prensner and Chinnaiyan, 2011; Zhou et al., 2015a; Zhou et al., 2015b; Arun et al., 2018). Recent studies showed that lncRNAs appear to be a critical regulator in the immune system (Chen et al., 2017; Wang and Zheng, 2018). However, a comprehensive analysis of lncRNAs changes induced by TLRs and their roles in diagnosis and prognosis in ESCA is still in its infancy.

In this study, we first examined the expression pattern of lncRNAs between 179 paired ESCA patients and normal tissues and identified 587 differentially expressed lncRNAs, implying their potential roles in ESCA development. By investigating the co-expression relationship between dysregulated IncRNAs and known TLR genes, we found that 357 of 587 differentially expressed lncRNAs are significantly correlated with at least one of TLR genes, suggesting that dysregulated expression of these 357 lncRNAs may be induced by TLR genes. A global network between TLR gene and lncRNAs was constructed, which provides a potential way to understand the mechanisms by which lncRNAs regulate TLR-driven responses in the innate immune system for ESCA development. To further explore potential clinical implication of TLRrelated lncRNAs in ESCA, we performed LASSO analysis for feature selection and identified four prognostic lncRNAs (AP000696.1, LINC00689, LINC00900, and AP000487.1) from the lists of 357 TLR-related lncRNAs. To accelerate the clinical application, these four TLR-related prognostic RNAs were integrated into a lncRNAs-based signature, which was capable of stratifying patients into high-risk and low-risk groups with significantly different OS in the discovery set. Further analysis in different independent patient sets also confirmed the robustness of the prognostic value of four-TLR-lncRNA signature in predicting OS of ESCA patients. Moreover, results of multivariate analysis in different patient sets indicated that the four-TLR-lncRNA signature is an independent factor after adjusted by other clinical factors (including age, gender, stage, and alcohol).

Of four lncRNAs biomarkers, AP000696.1 is essential in the development of ectoderm and epithelial cells, and may sever as prognostic biomarker (Li et al., 2017). It has been reported that LINC00900 is significantly up-regulated by all-trans-retinoic acid and down-regulated by vitamin D (Riege et al., 2017). LINC00689 has been observed to be deregulated expressed in ESCC. Furthermore, LINC00689 also was recently reported to be involved in osteosarcoma progression via the miR-655/SOC18 axis (Xing et al., 2020) and growth, metastasis and glycolysis of glioma cells by targeting miR-338-3p/PKM2 axis (Liu et al., 2019). In silico functional analysis demonstrated that co-expressed genes with the four-TLR-lncRNA signature tended to be enriched in TLR-related and cancer-related biological progress and pathways. For example, damage-associated molecules patterns (DAMPs) 
have been found to be ligands for TLRs. Recent studies have reported that the extracellular matrix (ECM)-driven DAMPs contributed to the activation of TLR4 signaling during the tumor progression (Kelsh and McKeown-Longo, 2013). The iNOS/Src/Fak axis has revealed critical roles for macrophages in TLR-mediated cell motility (Maa et al., 2011). Coordinate regulation of TLR-mediated arachidonic acid metabolism in macrophages has been reported to be involved in a variety of innate immune responses (Ruiperez et al., 2009). Although in silico functional analysis revealed the functional implication of the four-TLR-lncRNA signature in TLR pathways, further experimental studies were needed for verifying and deciphering regulatory mechanisms between these identified lncRNAs and TLR pathways in ESCA. Another limitation is that the prognostic value of the four-TLR-lncRNA signature was analyzed only in public datasets of ESCA, therefore, further retrospective studies or prospective clinical trials are needed.

In conclusion, in this study, we investigated functional roles of lncRNAs in TLR signaling pathways and their effects on the outcome of ESCA patients. The four-lncRNA signature induced by TLRs identified in this study represents a promising biomarker for outcome prediction and provides new candidate targets for therapeutic intervention via targeting lncRNAs and their TLR partners in ESCA patients.

\section{REFERENCES}

Arun, G., Diermeier, S. D., and Spector, D. L. (2018). Therapeutic targeting of long Non-coding RNAs in cancer. Trends Mol. Med. 24, 257-277. doi: 10.1016/j. molmed.2018.01.001

Bao, S., Zhao, H., Yuan, J., Fan, D., Zhang, Z., Su, J., et al. (2019). Computational identification of mutator-derived lncRNA signatures of genome instability for improving the clinical outcome of cancers: a case study in breast cancer. Brief. Bioinform. bbz118. doi: 10.1093/bib/bbz118

Cen, X., Liu, S., and Cheng, K. (2018). The role of toll-like receptor in inflammation and tumor immunity. Front. Pharmacol. 9:878. doi: 10.3389/fphar.2018.00878

Chen, Y. G., Satpathy, A. T., and Chang, H. Y. (2017). Gene regulation in the immune system by long noncoding RNAs. Nat. Immunol. 18, 962-972. doi: 10.1038/ni.3771

Djebali, S., Davis, C. A., Merkel, A., Dobin, A., Lassmann, T., Mortazavi, A., et al. (2012). Landscape of transcription in human cells. Nature 489, 101-108. doi: $10.1038 /$ nature 11233

D'Journo, X. B., and Thomas, P. A. (2014). Current management of esophageal cancer. J. Thorac. Dis. 6(Suppl. 2), S253-S264. doi: 10.3978/j.issn.2072-1439. 2014.04.16

Garrido, P., Conde, E., de Castro, J., Gomez-Roman, J. J., Felip, E., Pijuan, L., et al. (2020). Updated guidelines for predictive biomarker testing in advanced nonsmall-cell lung cancer: a national consensus of the spanish society of pathology and the Spanish society of medical oncology. Clin. Transl. Oncol. 22, 989-1003. doi: 10.1007/s12094-019-02218-4

Han Li, C., and Chen, Y. (2015). Small and long Non-coding RNAs: novel targets in perspective cancer therapy. Curr. Genomics 16, 319-326. doi: 10.2174/ 1389202916666150707155851

Hartwell, M. J., Ozbek, U., Holler, E., Renteria, A. S., Major-Monfried, H., Reddy, P., et al. (2018). An early-biomarker algorithm predicts lethal graftversus-host disease and survival. JCI Insight 3:e124015. doi: 10.1172/jci.insight. 124015

Jain, S., and Dhingra, S. (2017). Pathology of esophageal cancer and Barrett's esophagus. Ann. Cardiothorac. Surg. 6, 99-109. doi: 10.21037/acs.2017.03.06

Kawasaki, T., and Kawai, T. (2014). Toll-like receptor signaling pathways. Front. Immunol. 5:461. doi: 10.3389/fimmu.2014.00461

\section{DATA AVAILABILITY STATEMENT}

Publicly available datasets were analyzed in this study. This data can be found here: Clinical information of ESCA patients was downloaded from GSE53625 (https://www.ncbi.nlm.nih. gov/geo/query/acc.cgi?acc=GSE53625) and TCGA (https://www. cancer.gov/).

\section{AUTHOR CONTRIBUTIONS}

DZ and XW conceived and designed the experiments. JL, YW, YC, and RX performed the experiments and analyzed the data. JL and YW wrote the manuscript. All authors read and approved the final manuscript.

\section{SUPPLEMENTARY MATERIAL}

The Supplementary Material for this article can be found online at: https://www.frontiersin.org/articles/10.3389/fcell.2020.00649/ full\#supplementary-material

TABLE S1 | Lists of differentially expressed IncRNAs between 179 paired ESCA patients and normal tissues.

Kelsh, R. M., and McKeown-Longo, P. J. (2013). Topographical changes in extracellular matrix: activation of TLR4 signaling and solid tumor progression. Trends Cancer Res. 9, 1-13.

Koch, L. (2017). Functional genomics: screening for lncRNA function. Nat. Rev. Genet. 18:70. doi: 10.1038/nrg.2016.168

Li, J., Chen, Z., Tian, L., Zhou, C., He, M. Y., Gao, Y., et al. (2014). LncRNA profile study reveals a three-lncRNA signature associated with the survival of patients with oesophageal squamous cell carcinoma. Gut 63, 1700-1710. doi: 10.1136/gutjnl-2013-305806

Li, J., and Liu, C. (2019). Coding or noncoding, the converging concepts of RNAs. Front. Genet. 10:496. doi: 10.3389/fgene.2019.00496

Li, Z., Yao, Q., Zhao, S., Wang, Y., Li, Y., and Wang, Z. (2017). Comprehensive analysis of differential co-expression patterns reveal transcriptional dysregulation mechanism and identify novel prognostic lncRNAs in esophageal squamous cell carcinoma. Onco Targets Ther. 10, 3095-3105. doi: 10.2147/OTT.S135312

Liu, X., Zhang, M., Ying, S., Zhang, C., Lin, R., Zheng, J., et al. (2017). Genetic alterations in esophageal tissues from squamous dysplasia to carcinoma. Gastroenterology 153, 166-177. doi: 10.1053/j.gastro.2017.03.033

Liu, X., Zhu, Q., Guo, Y., Xiao, Z., Hu, L., and Xu, Q. (2019). LncRNA LINC00689 promotes the growth, metastasis and glycolysis of glioma cells by targeting miR338-3p/PKM2 axis. Biomed. Pharmacother. 117:109069. doi: 10.1016/j.biopha. 2019.109069

Maa, M. C., Chang, M. Y., Li, J., Li, Y. Y., Hsieh, M. Y., Yang, C. J., et al. (2011). The iNOS/Src/FAK axis is critical in Toll-like receptor-mediated cell motility in macrophages. Biochim. Biophys. Acta 1813, 136-147. doi: 10.1016/j.bbamcr. 2010.09.004

Mawhinney, M. R., and Glasgow, R. E. (2012). Current treatment options for the management of esophageal cancer. Cancer Manag. Res. 4, 367-377. doi: 10.2147/CMAR.S27593

Murphy, M. B., and Medvedev, A. E. (2016). Long noncoding RNAs as regulators of Toll-like receptor signaling and innate immunity. J. Leukoc. Biol. 99, 839-850. doi: 10.1189/jlb.2RU1215-575R

Napier, K. J., Scheerer, M., and Misra, S. (2014). Esophageal cancer: a review of epidemiology, pathogenesis, staging workup and treatment modalities. World J. Gastrointest. Oncol. 6, 112-120. doi: 10.4251/wjgo.v6.i5.112 
Penn-Nicholson, A., Hraha, T., Thompson, E. G., Sterling, D., Mbandi, S. K., Wall, K. M., et al. (2019). Discovery and validation of a prognostic proteomic signature for tuberculosis progression: a prospective cohort study. PLoS Med. 16:e1002781. doi: 10.1371/journal.pmed.1002781

Perry, R. B., and Ulitsky, I. (2016). The functions of long noncoding RNAs in development and stem cells. Development 143, 3882-3894. doi: 10.1242/dev. 140962

Prensner, J. R., and Chinnaiyan, A. M. (2011). The emergence of lncRNAs in cancer biology. Cancer Discov. 1, 391-407. doi: 10.1158/2159-8290.CD-110209

Qiu, M. T., Hu, J. W., Yin, R., and Xu, L. (2013). Long noncoding RNA: an emerging paradigm of cancer research. Tumour. Biol. 34, 613-620. doi: 10.1007/s13277013-0658-6

Riege, K., Holzer, M., Klassert, T. E., Barth, E., Brauer, J., Collatz, M., et al. (2017). Massive effect on LncRNAs in human monocytes during fungal and bacterial infections and in response to vitamins A and D. Sci. Rep. 7:40598. doi: $10.1038 /$ srep40598

Rinn, J. L., and Chang, H. Y. (2012). Genome regulation by long noncoding RNAs. Annu. Rev. Biochem. 81, 145-166.

Ruiperez, V., Astudillo, A. M., Balboa, M. A., and Balsinde, J. (2009). Coordinate regulation of TLR-mediated arachidonic acid mobilization in macrophages by group IVA and group V phospholipase A2s. J. Immunol. 182, 3877-3883. doi: 10.4049/jimmunol.0804003

Rustgi, A. K., and El-Serag, H. B. (2014). Esophageal carcinoma. N. Engl. J. Med. 371, 2499-2509. doi: 10.1056/NEJMra1314530

Sheyhidin, I., Nabi, G., Hasim, A., Zhang, R. P., Ainiwaer, J., Ma, H., et al. (2011). Overexpression of TLR3, TLR4, TLR7 and TLR9 in esophageal squamous cell carcinoma. World J. Gastroenterol. 17, 3745-3751. doi: 10.3748/wjg.v17.i32. 3745

Siegel, R. L., Miller, K. D., and Jemal, A. (2020). Cancer statistics, 2020. CA Cancer J. Clin. 70, 7-30. doi: 10.3322/caac. 21590

So, E. Y., and Ouchi, T. (2010). The application of Toll like receptors for cancer therapy. Int. J. Biol. Sci. 6, 675-681. doi: 10.7150/ijbs.6.675

Sun, J., Zhang, Z., Bao, S., Yan, C., Hou, P., Wu, N., et al. (2020). Identification of tumor immune infiltration-associated lncRNAs for improving prognosis and immunotherapy response of patients with non-small cell lung cancer. J. Immunother. Cancer 8:e00110. doi: 10.1136/jitc-2019-000110

Tang, W. W., Wu, Q., Li, S. Q., Tong, Y. S., Liu, Z. H., Yang, T. X., et al. (2015). Implication of lncRNAs in pathogenesis of esophageal cancer. Onco Targets Ther. 8, 3219-3226. doi: 10.2147/OTT.S87856

Tanzer, M., Liebl, M., and Quante, M. (2013). Molecular biomarkers in esophageal, gastric, and colorectal adenocarcinoma. Pharmacol. Ther. 140, 133-147. doi: 10.1016/j.pharmthera.2013.06.005

The Cancer Genome Atlas Research Network (2017). Integrated genomic characterization of oesophageal carcinoma. Nature 541, 169-175. doi: 10.1038/ nature20805

Wald, O., Smaglo, B., Mok, H., and Groth, S. S. (2017). Future directions in esophageal cancer therapy. Ann. Cardiothorac. Surg. 6, 159-166. doi: 10.21037/ acs.2017.02.01
Wang, Z., and Zheng, Y. (2018). lncRNAs regulate innate immune responses and their roles in macrophage polarization. Mediators Inflamm. 2018:8050956. doi: 10.1155/2018/8050956

Xing, W., Xu, W. Y., Chang, L., Zhang, K., and Wang, S. R. (2020). SP1-induced IncRNA LINC00689 overexpression contributes to osteosarcoma progression via the miR-655/SOX18 axis. Eur. Rev. Med. Pharmacol. Sci. 24, 2205-2217. doi: 10.26355/eurrev_202003_20486

Yu, G., Wang, L. G., Han, Y., and He, Q. Y. (2012). clusterProfiler: an R package for comparing biological themes among gene clusters. OMICS 16, 284-287. doi: 10.1089/omi.2011.0118

Zhang, Z., Bao, S., Yan, C., Hou, P., Zhou, M., and Sun, J. (2020). Computational principles and practice for decoding immune contexture in the tumor microenvironment. Brief. Bioinform. bbaa075. doi: 10.1093/bib/bbaa075

Zhou, M., Guo, M., He, D., Wang, X., Cui, Y., Yang, H., et al. (2015a). A potential signature of eight long non-coding RNAs predicts survival in patients with non-small cell lung cancer. J. Transl. Med. 13:231. doi: 10.1186/s12967-015-0 556-3

Zhou, M., Zhao, H., Wang, Z., Cheng, L., Yang, L., Shi, H., et al. (2015b). Identification and validation of potential prognostic lncRNA biomarkers for predicting survival in patients with multiple myeloma. J. Exp. Clin. Cancer Res. 34:102. doi: 10.1186/s13046-015-0219-5

Zhou, M., Hu, L., Zhang, Z., Wu, N., Sun, J., and Su, J. (2018a). Recurrenceassociated long Non-coding RNA Signature for determining the risk of recurrence in patients with colon cancer. Mol. Ther. Nucleic Acids 12, 518-529. doi: 10.1016/j.omtn.2018.06.007

Zhou, M., Zhang, Z., Zhao, H., Bao, S., Cheng, L., and Sun, J. (2018b). An immunerelated six-lncRNA signature to improve prognosis prediction of glioblastoma multiforme. Mol. Neurobiol. 55, 3684-3697. doi: 10.1007/s12035-017-0572-9

Zhou, M., Zhang, Z., Zhao, H., Bao, S., and Sun, J. (2018c). A novel lncRNA-focus expression signature for survival prediction in endometrial carcinoma. BMC Cancer 18:39. doi: 10.1186/s12885-017-3983-0

Zhou, M., Zhang, Z., Bao, S., Hou, P., Yan, C., Su, J., et al. (2020). Computational recognition of IncRNA signature of tumor-infiltrating B lymphocytes with potential implications in prognosis and immunotherapy of bladder cancer. Brief. Bioinform. bbaa047. doi: 10.1093/bib/bbaa047

Conflict of Interest: The authors declare that the research was conducted in the absence of any commercial or financial relationships that could be construed as a potential conflict of interest.

The reviewer XC declared a shared affiliation with the authors to the handling editor at time of review.

Copyright (c) $2020 \mathrm{Liu}$, Wang, Chu, Xu, Zhang and Wang. This is an open-access article distributed under the terms of the Creative Commons Attribution License (CC BY). The use, distribution or reproduction in other forums is permitted, provided the original author(s) and the copyright owner(s) are credited and that the original publication in this journal is cited, in accordance with accepted academic practice. No use, distribution or reproduction is permitted which does not comply with these terms. 